\title{
Pilot study of intraoperative ultrasound-guided instrument placement in nerve transection surgery for peripheral nerve pain syndromes
}

\author{
P. Troy Henning, DO, ${ }^{1}$ Thomas J. Wilson, MD, ${ }^{2}$ Matthew Willsey, MD, MS, ${ }^{3}$ \\ Jessin K. John, MD, PhD, ${ }^{4}$ Miriana Popadich, MSN, ${ }^{3}$ and Lynda J. S. Yang, MD, PhD ${ }^{3}$ \\ Departments of ${ }^{1}$ Physical Medicine \& Rehabilitation and ${ }^{3}$ Neurosurgery, University of Michigan, Ann Arbor, Michigan; \\ 2Department of Neurosurgery, Mayo Clinic, Rochester, Minnesota; and ${ }^{4}$ Department of Neurosurgery, Henry Ford Health \\ System, Detroit, Michigan
}

\begin{abstract}
Surgical transection of sensory nerves in the treatment of intractable neuropathic pain is a commonly performed procedure. At times these cases can be particularly challenging when encountering obese patients, when targeting deeper nerves or those with a variable branching pattern, or in the case of repeat operations. In this case series, the authors describe their experience with ultrasound-guided surgical instrument placement during transection of a saphenous nerve in the region of prior vascular surgery in 1 patient and in the lateral femoral cutaneous nerve in 2 obese patients. The authors also describe this novel technique and provide pilot data that suggests ultrasound-assisted surgery may allow for complex cases to be completed in an expedited fashion through smaller incisions.
\end{abstract}

https://thejns.org/doi/abs/10.3171/2017.1.FOCUS16438

KEY WORDS nerve transection; peripheral nerve; surgical instrument; ultrasound guidance

$\mathrm{T}$ RaDiTIONALly, localization of peripheral nerves at the time of surgery has been based on anatomical surface landmarks. ${ }^{8,13}$ For superficial nerves, a small incision with minimal tissue dissection may be possible. In contrast, deeper nerves often require a large surgical exposure with extensive tissue dissection. Additionally, variations in neural anatomy pose challenges, which may lead to prolonged surgical time to adequately localize the nerve and may also lead to poor outcomes when variations in nerve branching are not recognized..$^{1,3}$ Options for preoperative evaluation of peripheral nerve lesions include imaging-typically MRI and/or ultrasonography-as well as electromyographic studies. ${ }^{2,17}$ However, these studies still do not help the surgeon localize the nerve in an expedited fashion at the time of surgery. Prior case reports have described localizing nerves with preoperative staining of the nerve and peripheral tissue track with methylene blue dye, ${ }^{11}$ or localizing by use of guidewires. ${ }^{15}$ One review described basic ultrasound-guided surgical techniques including needle-based hydrodissection and use of typical endoscopic surgical instruments for tissue dissection. ${ }^{12}$ To the best of our knowledge, there are no prior case reports describing the use of ultrasonography in the operat- ing room to help directly guide the placement of surgical instruments to aid localization of nerves for transection. Our case series also reports data to show how ultrasoundguided surgery may reduce both operative time and the amount of tissue dissection needed for these commonly performed procedures. In this paper we discuss our experience with the use of this novel technique to transect 1 saphenous and 2 lateral femoral cutaneous nerves (LFCNs) quickly through small incisions.

\section{Methods}

A series of 3 patients with peripheral nerve pain syndromes underwent peripheral nerve transection. The planned nerve for transection was the saphenous nerve in 1 case and the LFCN in 2 cases.

In each case, after induction of general anesthesia, the planned operative site was prepared and draped in the usual sterile fashion. Next, ultrasound guidance was used to place a surgical instrument at the planned site of transection (P.T.H.). The specific technique used in each case is provided below. A Philips CX30 portable ultrasonography machine (Philips Corp.) with a 12-4 linear array 


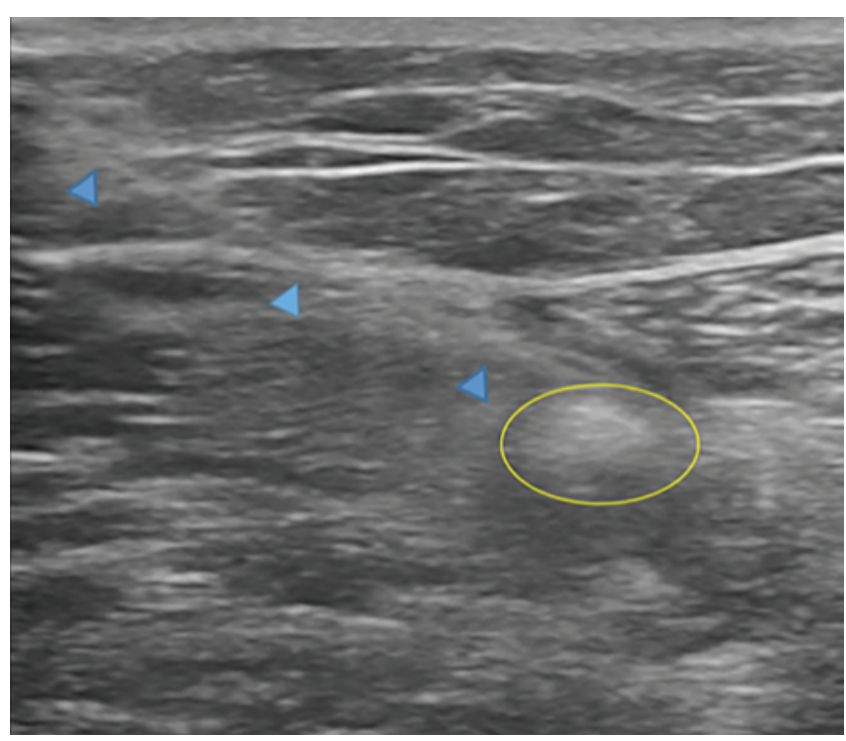

FIG. 1. Case 1. Needle placement adjacent to the saphenous nerve (oval). Arrowheads indicate the needle. Right $=$ posterior, left $=$ anterior, upper $=$ superficial, lower $=$ deep .

transducer was used for ultrasound guidance. The nerve of interest was then identified and transected by the lead surgeon (L.J.S.Y.).

Data collected for each case included time of ultrasound visualization of the nerve, time of surgical transection, ending time of surgical wound closure, length of the counter incision used for introduction of the clamp, length of the primary incision through which the nerve transection was performed, and body mass index (BMI) of the patient.

\section{Results}

\section{Case 1: Saphenous Nerve Transection}

A 64-year-old man with a BMI of $29.12 \mathrm{~kg} / \mathrm{m}^{2}$ and a medical history significant for peripheral vascular disease, tobacco use, and diabetes mellitus underwent a right femoral above-the-knee popliteal bypass with graft for lifestyle-limiting claudication. The grafting was complicated by clotting and infection that led to removal of a portion of the graft. Following this surgery, his pain persisted but now also included painful tingling along the medial knee and lower medial leg. He subsequently underwent removal of the remaining graft in the groin region, with patch angioplasty using a saphenous vein performed at that time. Unfortunately, this still did not alleviate his pain. Gabapentin was initiated and titrated to $600 \mathrm{mg} 3$ times daily without a reduction in symptoms. The patient was then referred for evaluation by physical medicine and rehabilitation, where it was determined that his pain symptoms and physical examination (a positive Tinel sign along the saphenous nerve at the distal thigh incision site) were most consistent with saphenous neuropathy, likely from the initial removal of the lower portion of the vascular graft. A sonographically guided saphenous nerve block performed in the clinic with $2 \mathrm{ml}$ of local anesthetic injected around the nerve at the level of the distal one-third of the thigh completely alleviated his pain. He began receiving amitriptyline hydrochloride that was titrated to $25 \mathrm{mg}$ twice daily. While this treatment reduced his pain, he could not tolerate the medication because of sedation and dry mouth. He then elected to undergo nerve transection.

Surgery was performed with ultrasound-guided assistance. After the typical anatomical landmarks were identified and marked on the patient's skin, he was properly anesthetized, cleansed, and draped. Surgery began with sonographic localization of the saphenous nerve between the overlying sartorius and deep vastus medialis muscles. A 22-gauge, 3.5-inch needle was sonographically guided down to the nerve, where $10 \mathrm{ml}$ of sterile $0.9 \%$ normal saline was injected to hydrodissect the nerve away from adjacent tissue to improve visualization (Fig. 1). Then, a 4-mm incision was made at the prior needle entry point. Next, under sonographic visualization a scalpel was used to make a small opening in the thigh fascia. A pituitary forceps was introduced into the wound and sonographically guided down to the saphenous nerve, which was then clamped in the forceps. A 26-mm incision was made along the typical course of the saphenous nerve, based on surface anatomical landmarks. The tip of the forceps could be palpated through the exposed site. Dissection was carefully carried down to the forceps where the nerve was visualized within the clamp. The nerve was secured and transected, and the wounds were closed in the typical fashion. Pathology confirmed the presence of neural tissue. The duration between ultrasound visualization of the nerve and nerve transection was 10 minutes. Total duration of the case from ultrasound visualization to completion of wound closure was 17 minutes (Table 1). Pre- and postoperative pain were assessed in a retrospective fashion using WongBaker FACES Pain Rating Scale (http://wongbakerfaces. org/) and a 100-mm visual analog scale (VAS). Fortythree days after surgery this patient reported a reduction in the Wong-Baker FACES Scale score from 8 to 4 and the VAS score decreased from 89 to 24 (Table 2).

\section{Case 2: LFCN Transection}

A 74-year-old woman with a medical history of coro-

TABLE 1. Data collected at time of surgery for the 3 cases presented

\begin{tabular}{|c|c|c|c|c|c|c|c|}
\hline \multirow[b]{2}{*}{ Case No. } & \multicolumn{3}{|c|}{ Clock Time } & \multirow{2}{*}{$\begin{array}{l}\text { Total Duration } \\
\text { (mins) }\end{array}$} & \multirow{2}{*}{$\begin{array}{l}\text { Clamp Incision } \\
\text { Length }(\mathrm{mm})\end{array}$} & \multirow{2}{*}{$\begin{array}{l}\text { Primary Incision } \\
\text { Length (mm) }\end{array}$} & \multirow{2}{*}{$\begin{array}{c}\text { BMl } \\
\left(\mathrm{kg} / \mathrm{m}^{2}\right)\end{array}$} \\
\hline & US Visualization & Nerve Transection & Wound Closure Completed & & & & \\
\hline 1 & 9:08 & $9: 18$ & $9: 25$ & 17 & 4 & 26 & 29.12 \\
\hline 2 & $10: 37$ & $10: 51$ & $10: 59$ & 22 & 3 & 30 & 30.16 \\
\hline 3 & $12: 14$ & $12: 21$ & $12: 33$ & 19 & 3 & 30 & 38.70 \\
\hline
\end{tabular}

US = ultrasound. 
TABLE 2. Pain score data collected postoperatively

\begin{tabular}{cccccc}
\hline & \multicolumn{2}{c}{ Preop } & & \multicolumn{2}{c}{ Postop } \\
\cline { 2 - 3 } \cline { 5 - 6 } Case No. & $\begin{array}{c}\text { Wong-Baker } \\
\text { FACES Scale }\end{array}$ & VAS & & $\begin{array}{c}\text { Wong-Baker } \\
\text { FACES Scale }\end{array}$ & VAS \\
\hline 1 & 8 & 89 & 4 & 24 \\
\hline 2 & 6 & 61 & 6 & 30 \\
\hline 3 & 6 & 68 & 4 & 32 \\
\hline
\end{tabular}

Pre- and postoperative data were collected 43 days after surgery for Case 1 and 49 days after surgery for Cases 2 and 3. Wong-Baker FACES Scale range $0-10$, VAS range $0-100 \mathrm{~mm}$.

nary artery disease, stroke, hypertension, hypothyroidism, and obesity (BMI $30.16 \mathrm{~kg} / \mathrm{m}^{2}$ ) was examined in the physical medicine and rehabilitation clinic for a several-year history of right lateral hip pain. On physical examination, she reported tenderness to palpation along the right greater trochanter. She had no pain with examination of the hip joint. A lumbar Spurling maneuver was negative, as was a femoral nerve stretch test. She reported pain with light touch along the right anterolateral thigh. Sensory, tendon reflex, and motor examinations were otherwise normal and symmetric. Prior imaging included MRI of the lumbar spine, which revealed multilevel spondylosis without significant central or neuroforaminal stenosis. MRI of the hip revealed arthritis of the hip joint and gluteal tendinopathy. She was initially treated for gluteal tendinopathy with physical therapy, trochanteric bursa injection, and nitroglycerin patches. She was not responding to this treatment regimen, and upon further questioning, she reported painful tingling along the right anterolateral thigh. She underwent a sonographically guided perineural corticosteroid injection along the LFCN at the level of the anterior superior iliac spine with $2 \mathrm{ml}$ of $1 \%$ lidocaine and $1 \mathrm{ml}$ of $40 \mathrm{mg} / \mathrm{ml}$ methylprednisolone. She reported immediate relief of the thigh pain within 10 minutes after injection. She was treated with gabapentin $600 \mathrm{mg} 3$ times daily for 3 months, but could not tolerate this regimen because of its sedating qualities. The decision was made to pursue operative transection of the LFCN using ultrasound guidance.

After induction of general anesthesia, the typical anatomical landmarks were identified and marked on the patient's skin. She was then prepared and draped in the usual fashion. Surgery began with sonographic localization of the LFCN located in a fascial plane between the lateral-lying tensor fascia and medial sartorius muscles. A 25-gauge, 1.5-inch needle was sonographically guided down to the nerve where $10 \mathrm{ml}$ of sterile $0.9 \%$ normal saline was injected to hydrodissect the nerve away from adjacent tissue to improve visualization. A 3-mm incision was then made at the prior needle entry point. Next, a scalpel was inserted under sonographic visualization to make a small opening in the thigh fascia. A pituitary forceps was introduced into the wound and sonographically guided down to the LFCN, which was then clamped in the forceps (Figs. 2 and 3). A 3-cm incision (Table 1) was made along the typical course of the LFCN according to anatomical landmarks. Forceps were used to deliver the nerve up to the exposed site, allowing visualization of the

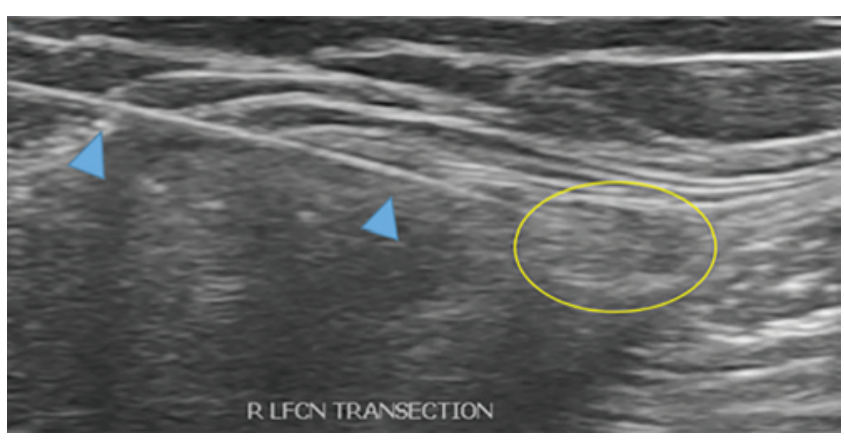

FIG. 2. Case 2. Pituitary forceps around the right LFCN. Arrowheads denote pituitary forceps, oval encircles right LFCN. Right = medial, left = lateral, upper = superficial, lower = deep.

nerve within the clamp. The nerve was secured and transected, and the wounds were closed in the typical fashion. Pathology confirmed the presence of neural tissue. The time from ultrasound visualization of the nerve to transection was 14 minutes. Total case time from ultrasound visualization to complete closure of the wound was 22 minutes (Table 1). Forty-nine days after surgery this patient reported no change in her Wong-Baker FACES Scale score, but her VAS score changed from 61 to 30 (Table 2).

\section{Case 3: LFCN Transection}

A 71-year-old woman with a medical history significant for obesity (BMI $38.70 \mathrm{~kg} / \mathrm{mm}^{2}$ ), diabetes mellitus, and a prior spine fusion at L3-4 presented to the peripheral nerve surgery clinic with a several-year history of left anterolateral thigh pain. She had been treated with gabapentin but could only tolerate $100 \mathrm{mg}$ twice daily. Physical examination revealed painful light touch along the anterolateral thigh and an otherwise normal neurological examination. Electrodiagnostic testing that day did not reveal evidence of lumbosacral radiculopathy or peripheral neuropathy. She underwent a sonographically guided perineural injection with $2 \mathrm{ml}$ of $2 \%$ lidocaine and $1 \mathrm{ml}$ of 40 $\mathrm{mg} / \mathrm{ml}$ triamcinolone acetonide around the left LFCN at the level of the anterior superior iliac spine. She reported $100 \%$ relief of pain within 10 minutes after the injection. She elected to undergo ultrasound-assisted nerve transection, which was performed in a similar fashion to Case 2. As described in Case 2, a 3-cm incision (Table 1) was made along the typical path for the LFCN based on anatomical landmarks. As in Case 2, the forceps were used to deliver the nerve into the wound. Again, the nerve was visualized within the clamp, secured and transected proximal to the forceps, followed by closure of the wound in a typical fashion. Pathology confirmed the presence of nerve tissue. The duration from ultrasound visualization to transection of the nerve was 7 minutes. Total case time from ultrasound visualization to complete wound closure was 19 minutes (Table 1). Forty-nine days after surgery this patient reported a reduction in the Wong-Baker FACES Scale score from 6 to 4 and the VAS score decreased from 68 to 32 (Table 2).

\section{Discussion}

To our knowledge, this is the first reported case series 


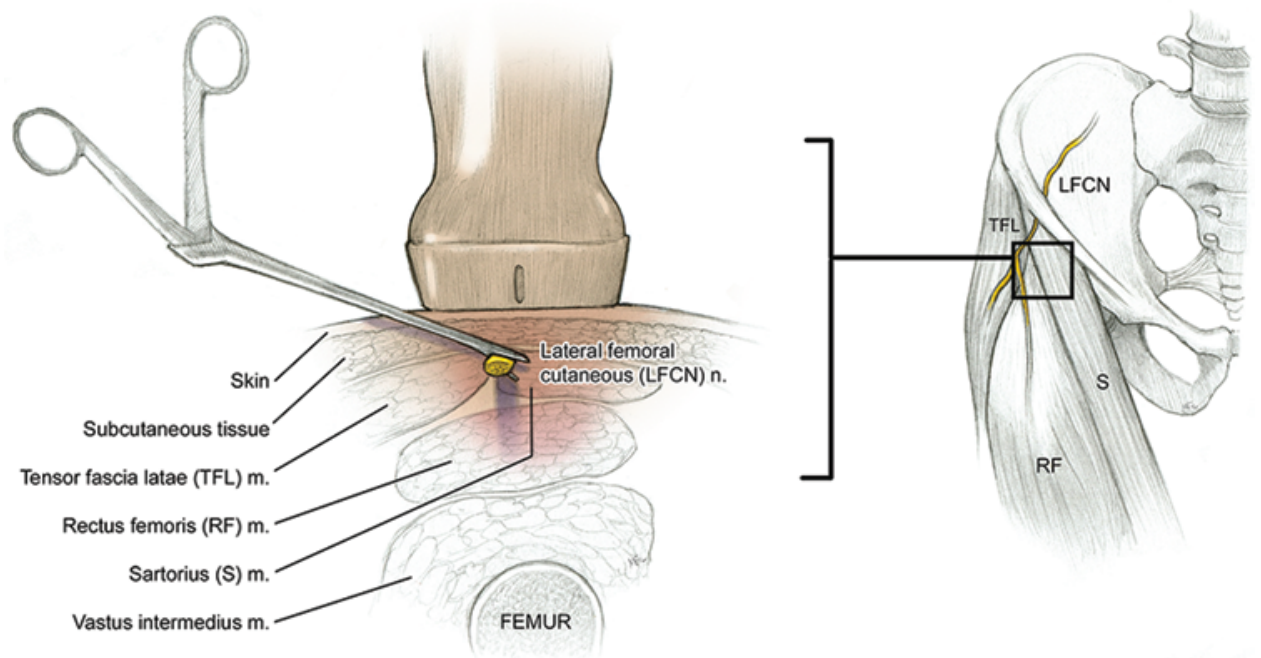

FIG. 3. Illustration depicting ultrasound-guided placement of the forceps around the LFCN located along the proximal thigh between the sartorius and tensor fascia latae muscles. Right $=$ medial, left = lateral, upper = superficial, lower $=$ deep, $\mathrm{m} .=$ muscle, $\mathrm{n} .=$ nerve. Copyright Megan Foldenauer. Published with permission.

describing the use of real-time ultrasound-assisted peripheral nerve surgery in which a surgical instrument was used to isolate the nerve and quickly direct the surgeon to the nerve through a relatively small surgical exposure. While in some instances short surgical times and small incisions may be possible to localize LFCNs and saphenous nerves using traditional landmark-based methods, the majority of cases involve a much longer length of time and larger incisions to adequately localize the nerve. This is particularly true when variant anatomy is present or-based on our experience-when repeat operations are performed and significant scar tissue is present.,6,9 However, using intraoperative ultrasonography, our longest case duration was 22 minutes with a wound closure length of $3 \mathrm{~cm}$. We expect these numbers to be even further reduced with increasing case volume and experience with this technique.

While not reflected in our presentation of these cases, a shorter operative duration will surely lower the risks associated with these types of procedures. Smaller incisions and less tissue dissection will reduce the potential creation of empty space and therefore reduce the risk of postoperative complications such as hematoma and deep tissue infections. Furthermore, the risk of surgical site infection has been correlated with operative duration. By decreasing the operative time, we will also likely decrease the risk of surgical site infection..$^{10,16}$ In addition to these benefits, this technique may also prove to be cost-effective. In an era in which there is increasing pressure to reduce health care costs, minimizing operative time can help contribute to reduced costs. At our institution, the charge for 1 minute in the operating room ranges from $\$ 65$ to $\$ 92$. As demonstrated by the short operative times, this technique has the potential to provide significant cost savings, given that a typical non-ultrasound-assisted case averages 2.9 hours.

The technique described here provides several advantages. First, ultrasound can simultaneously be used to identify variant anatomy, including variations in branching patterns that, if not accounted for, may provide suboptimal results, and then to localize the nerve of interest. Second, the nerve is localized under real-time visualization, and by clamping the nerve, identification is highly specific and also is unlikely to become dislodged, as can happen when guidewires are used. For superficial nerves, the clamp can also be used to deliver the nerve into the incision, which aids in further minimizing the size of incision required. Additionally, this methodology avoids any issues with neurotoxicity, ${ }^{7}$ allergic reaction, ${ }^{5}$ and the potential for permanent skin staining with the use of methylene blue. ${ }^{14}$ Finally, particularly in cases of meralgia paresthetica, patients are often obese. In these patients, identification of the LFCN can be particularly difficult and can require large incisions. We demonstrate that this technique can be used in obese patients with success, as in Case 3, a transection of the LFCN in a patient with a BMI of $38.70 \mathrm{~kg} / \mathrm{mm}^{2}$ through a $30-\mathrm{mm}$ primary incision in 19 minutes from ultrasound visualization to wound closure. While none of the patients experienced $100 \%$ pain relief upon their early postoperative visit, all of them demonstrated a significant reduction in pain as demonstrated in a $>20-\mathrm{mm}$ reduction in the VAS score. We believe complete pain relief in these patients would be unlikely from a single treatment intervention as they all had other nonneurological generators of pain.

This pilot study demonstrates proof of concept for this technique, but we acknowledge several limitations to the procedure. First, it may not be practical in all settings, given the lack of widespread availability of ultrasound equipment and skilled operators. However, the training of physicians outside of radiology and clinical integration of ultrasound for both diagnostic and interventional purposes is becoming more commonplace. Second, because this technique clamps the nerve, it can only be used in cases in which transection rather than decompression is planned, limiting this technique to pure sensory nerves. It is possible that this technique could be modified to place the clamp just deep or adjacent to the nerve of interest, clamping tissue juxtaposed to the nerve without clamping the nerve itself. This would still allow robust localization 
without damage to the nerve and may allow this technique to be used in additional circumstances.

While ultrasound-assisted surgery will not likely be needed for all peripheral nerve cases, we believe the technique described here has the potential to be a successful advancement in the field when applied to cases that involve deeper structures, nerves more likely to have a variable location or branching pattern, or in repeat cases. It also remains possible that this technique could be used for other peripheral nerve applications such as small nerve sheath tumors arising from small nerve branches that can generate considerable pain for patients and, when not palpable from the surface, can be extremely difficult to localize intraoperatively. Application of this technique may allow robust identification of these tumors. As we continue to develop this technique, we believe outcomes will continue to improve for the applications described here and that new applications will also likely become apparent.

\section{References}

1. Adkison DP, Bosse MJ, Gaccione DR, Gabriel KR: Anatomical variations in the course of the superficial peroneal nerve. J Bone Joint Surg Am 73:112-114, 1991

2. Bendszus M, Wessig C, Solymosi L, Reiners K, Koltzenburg M: MRI of peripheral nerve degeneration and regeneration: correlation with electrophysiology and histology. Exp Neurol 188:171-177, 2004

3. Carai A, Fenu G, Sechi E, Crotti FM, Montella A: Anatomical variability of the lateral femoral cutaneous nerve: findings from a surgical series. Clin Anat 22:365-370, 2009

4. de Ridder VA, de Lange S, Popta JV: Anatomical variations of the lateral femoral cutaneous nerve and the consequences for surgery. J Orthop Trauma 13:207-211, 1999

5. Dewachter P, Mouton-Faivre C, Tréchot P, Lleu JC, Mertes PM: Severe anaphylactic shock with methylene blue instillation. Anesth Analg 101:149-150, 2005

6. Gofeld M, Bristow SJ, Chiu S, Kliot M: Preoperative ultrasound-guided mapping of peripheral nerves. J Neurosurg 119:709-713, 2013

7. Hackett ER, Kline DG: Methylene blue. Surg Neurol 50:608-609, 1998

8. Haldeman CL, Baggott CD, Hanna AS: Intraoperative ultrasound-assisted peripheral nerve surgery. Neurosurg Focus 39(3):E4, 2015

9. Hospodar PP, Ashman ES, Traub JA: Anatomic study of the lateral femoral cutaneous nerve with respect to the ilioinguinal surgical dissection. J Orthop Trauma 13:17-19, 1999
10. Korinek AM, Golmard JL, Elcheick A, Bismuth R, van Effenterre R, Coriat P, et al: Risk factors for neurosurgical site infections after craniotomy: a critical reappraisal of antibiotic prophylaxis on 4,578 patients. Br J Neurosurg 19:155-162, 2005

11. Osorio JA, Breshears JD, Arnaout O, Simon NG, HastingsRobinson AM, Aleshi P, et al: Ultrasound-guided percutaneous injection of methylene blue to identify nerve pathology and guide surgery. Neurosurg Focus 39(3):E2, 2015

12. Pilecki Z, Koczy B, Mielnik M, Pilecki G, Dzielicki J, Jakubowski W: Basic dissecting techniques in ultrasoundguided surgery. J Ultrason 14:171-178, 2014

13. Quiñones-Hinojosa A (ed): Schmidek and Sweet: Operative Neurosurgical Techniques, ed 6. Philadelphia: Saunders Elsevier, 2012

14. Stradling B, Aranha G, Gabram S: Adverse skin lesions after methylene blue injections for sentinel lymph node localization. Am J Surg 184:350-352, 2002

15. Thomas AJ, Bull MJ, Howard AC, Saleh M: Peri operative ultrasound guided needle localisation of amputation stump neuroma. Injury 30:689-691, 1999

16. Valentini LG, Casali C, Chatenoud L, Chiaffarino F, UbertiFoppa C, Broggi G: Surgical site infections after elective neurosurgery: a survey of 1747 patients. Neurosurgery 62:88-96, 2008

17. Zaidman CM, Seelig MJ, Baker JC, Mackinnon SE, Pestronk A: Detection of peripheral nerve pathology: comparison of ultrasound and MRI. Neurology 80:1634-1640, 2013

\section{Disclosures}

The authors report no conflict of interest concerning the materials or methods used in this study or the findings specified in this paper.

\section{Author Contributions}

Conception and design: Henning, Yang. Acquisition of data: all authors. Analysis and interpretation of data: all authors. Drafting the article: Henning, Wilson, Willsey. Critically revising the article: Henning, Yang. Reviewed submitted version of manuscript: all authors. Approved the final version of the manuscript on behalf of all authors: Henning. Study supervision: Yang.

\section{Correspondence}

P. Troy Henning, Department of Physical Medicine \& Rehabilitation, University of Michigan, 325 E Eisenhower Pkwy., Ste. 100, Ann Arbor, MI 48108. email: ptroy@med.umich.edu. 